\title{
INTRODUCTION
}

The Accelerator Test Facility (ATF) is a research facility located at KEK in Tsukuba, Japan. The ATF consists of an RF gun, a $1.3 \mathrm{GeV}$ electron linac, a damping ring (DR), and a beamline known as ATF2 [1,2]. At the end of the ATF2 beamline, a pair of powerful quadrupole magnets is used to focus the electron beam to the smallest size possible at a location known as the interaction point (IP). The beamline o is shown in more detail in Fig. 1.

ह The ATF 2 Collaboration has two goals. Goal 1 is the production of a $37 \mathrm{~nm}$ vertical beam spot size at the IP. Goal 2 2 is the stabilization of the vertical beam position at the same vocation to the nanometer level $[3,4]$.

The ATF is capable of generating multi-bunch trains by accumulating bunches in the damping ring over the course of several pulses and then extracting them in a single pulse. The Feedback On Nanosecond Timescales (FONT) group at the University of Oxford developed a low-latency ( $\sim 150 \mathrm{~ns})$ single-phase beam feedback system [5] as a prototype of the intra-train beam stabilisation system required for the interaction point of the ILC. Here, we report the results of a feedback system based on this technology to stabilize both the beam position and the trajectory angle in the ATF2. The corrections were applied in the vertical plane locally in the

early part of the ATF2 beamline so as to deliver a stable beam to the entrance of the final focus system.

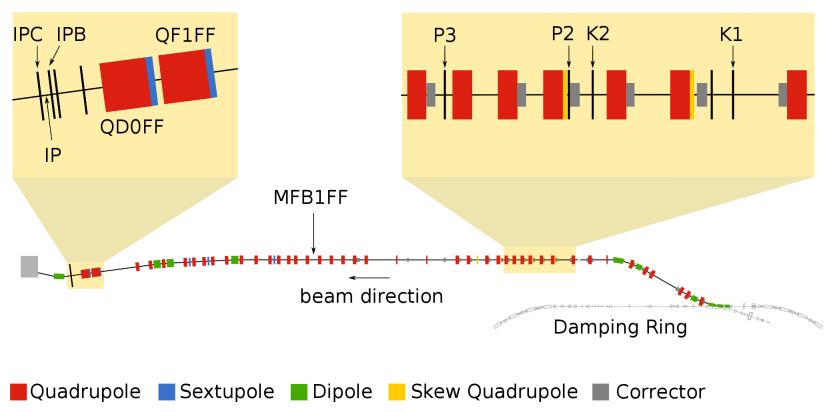

Figure 1: Schematic [6] of the ATF2 beamline showing the layout of components in the region of the FONT feedback system and at the IP.

\section{FEEDBACK SYSTEM}

The hardware of the feedback system is depicted schematically in Fig. 2. P2 and P3 are stripline BPMs. The voltage pulses induced on the top and bottom striplines by the passage of an electron bunch are processed using custom analogue electronic modules; the design of these BPMs and electronics has been previously reported [7]. The stripline voltage-difference signal $(\Delta)$ depends on both the vertical position of the bunch and its charge $Q$, while the stripline voltage-sum signal $(\Sigma)$ depends only on charge. The position of the bunch is derived from the ratio $\Delta / \Sigma .2016$ measurements with the system demonstrated a position resolution of $157 \pm 8 \mathrm{~nm}$ for a beam charge of $1.3 \mathrm{nC}\left(0.82 \times 10^{10} \mathrm{elec}-\right.$ trons/bunch) [8].

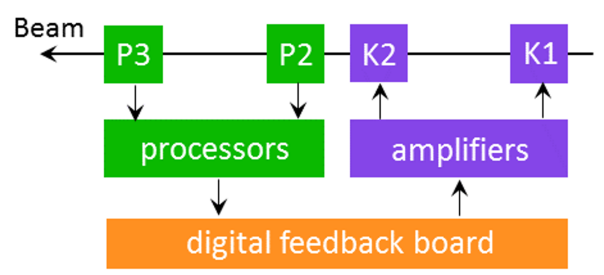

Figure 2: Schematic of the coupled-loop feedback system using BPMs P2 and P3 and kickers K1 and K2.

The processed BPM signals are input to a custom-made digital feedback ('FONT5') board [5,7]. The FONT5 board design features a Field-Programmable Gate Array (FPGA) along with nine analogue-to-digital converters and a pair of digital-to-analogue converters. The feedback algorithm runs 
on the FPGA and is able to calculate the appropriate kicker drive signals from the digitized BPM signals. The kicker drive signals are then amplified externally using bespoke ultra-fast amplifiers developed by TMD Technologies and applied to the stripline kickers K1 and K2. Further details of this system are reported in [9-13].

\section{RESULTS}

\section{Beam Stability}

The performance of the feedback system in terms of the beam stability is shown in Fig. 3. Bunch 1 provides the feedback input and its position is not corrected. Bunch 2 is well corrected by the feedback as shown by the substantial reduction in the position jitter seen at the two feedback BPMs: from $1.39 \pm 0.10 \mu \mathrm{m}$ to $0.34 \pm 0.02 \mu \mathrm{m}$ at $\mathrm{P} 2$ and from $0.93 \pm 0.07 \mu \mathrm{m}$ to $0.27 \pm 0.02 \mu \mathrm{m}$ at P3. The correction is limited by the resolution of BPMs P2 and P3, which was approximately $0.2 \mu \mathrm{m}$ for the bunch charge used $\left(0.45 \times 10^{10}\right.$ electrons). Three additional BPMs were used to witness the effect of the feedback further downstream: a stripline BPM MFB1FF located about halfway down the ATF2 beamline and a pair of cavity BPMs IPB and IPC [14] located at the IP. The correction factor at all three witness BPMs is consistent with the in-loop correction of roughly a factor of 4 .
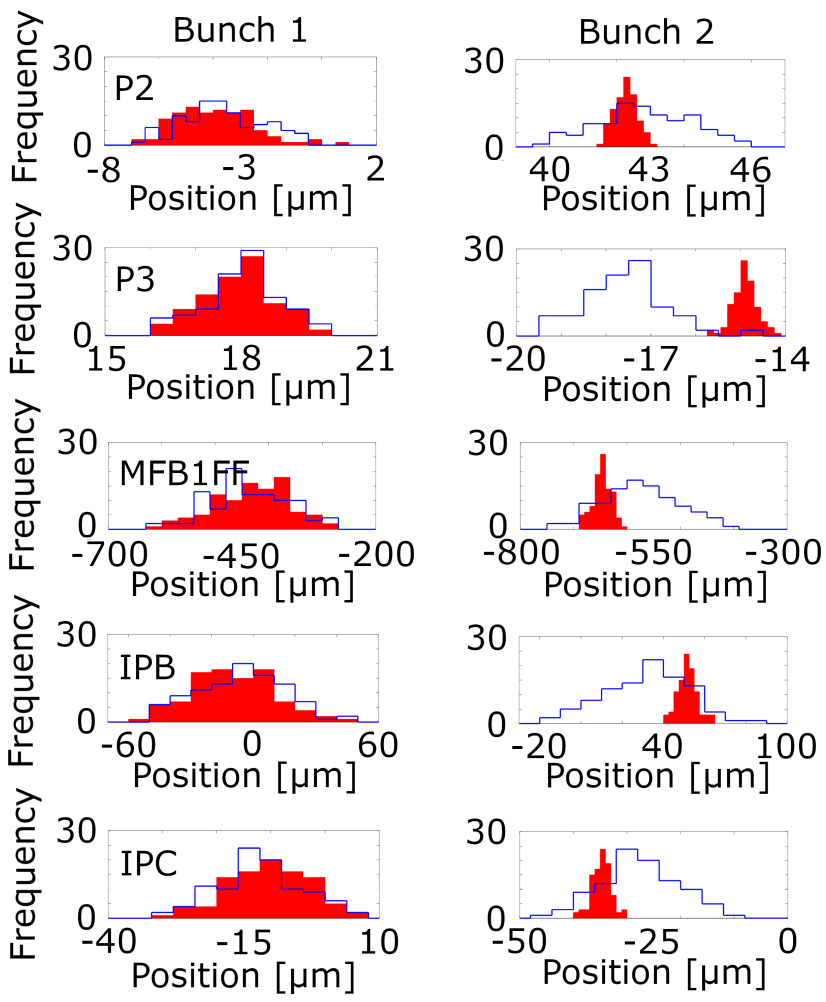

Figure 3: Distribution of position measured at each BPM (rows) for bunch 1 (left column) and bunch 2 (right column) with feedback off (outline) and on (filled).

The angular jitter of the bunch is calculated using the position measured at two BPMs and knowledge of how the beam propagates from one BPM to the other. The MAD model is used for the transfer matrix from P2 to P3; the measurements show a reduction in trajectory angle jitter from $1.08 \pm 0.08 \mu \mathrm{rad}$ to $0.24 \pm 0.02 \mu \mathrm{rad}$ at $\mathrm{P} 2$ and from $1.04 \pm 0.07 \mu \mathrm{rad}$ to $0.23 \pm 0.02 \mu \mathrm{rad}$ at P3. In the IP region the transfer matrix is trivially obtained as the beam propagates in a ballistic fashion from IPB to IPC; the trajectory angle jitter calculated from the cavity BPM measurements reduces from $154.5 \pm 11.0 \mu \mathrm{rad}$ to $37.4 \pm 2.7 \mu \mathrm{rad}$. The angles at $\mathrm{P} 3$ and in the IP region are shown in Fig. 4. The results show that the angular jitter of bunch 2 is also corrected by the feedback by about a factor of 4 , consistent with the position-correction analysis.
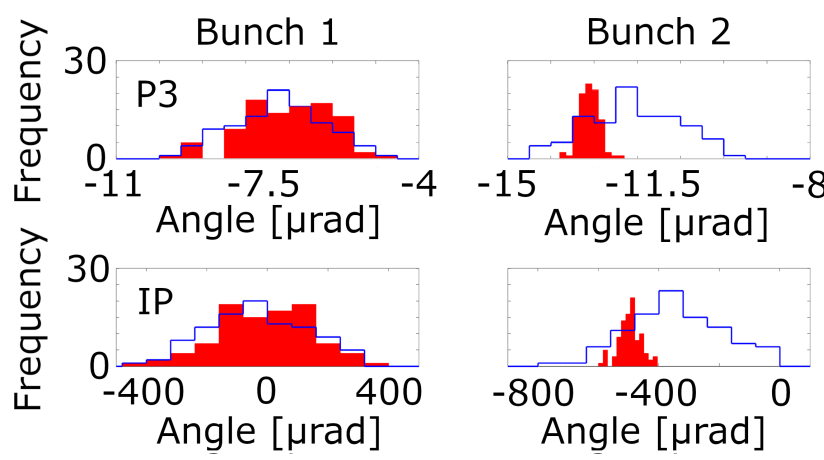

Figure 4: Distribution of angle at P3 (calculated from the position at $\mathrm{P} 2$ and $\mathrm{P} 3$ ) and in the IP region (calculated from the position at IPB and IPC) with feedback off (outline) and feedback on (filled).

The measured beam positions at $\mathrm{P} 2$ and $\mathrm{P} 3$ were extrapolated using the model to give predicted positions at the downstream BPMs. The predicted jitter values and respective correction factors are in good agreement with the direct measurements, implying that there are no major sources of additional beam jitter between the feedback kickers and the ATF2 final focus. The locally-measured beam angle jitter in the IP region is in good agreement with the model prediction.

The model can also be used to predict the beam position distribution at the focal point where the vertical beam position jitter is at a minimum; the results are shown in Fig. 5. With feedback off the predicted jitter is $2.9 \pm 0.2 \mathrm{~nm}$; with feedback operational, the equivalent jitter is $1.2 \pm 0.1 \mathrm{~nm}$. Therefore, to the extent that the beam transport model is correct, and assuming no additional jitter sources, it is possible that the FONT feedback system corrects the beam jitter at the focal point to the level of $1 \mathrm{~nm}$, thereby meeting the ATF2 beam stability goal. However, it is not possible with any known BPM technology to directly measure the beam position to the desired level of accuracy of order $1 \mathrm{~nm}$, so this prediction cannot be confirmed by direct measurement The best resolution of the cavity BPMs installed at the ATF2 IP achieved to date is c. $20 \mathrm{~nm}$ [15].

\section{Beam Size}

A nanometer-resolution IP beam size monitor (IPBSM) is installed at the ATF2 IP [16]. The device works by splitting

(1)
. 음 吾 西

\section{政}

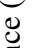
. 

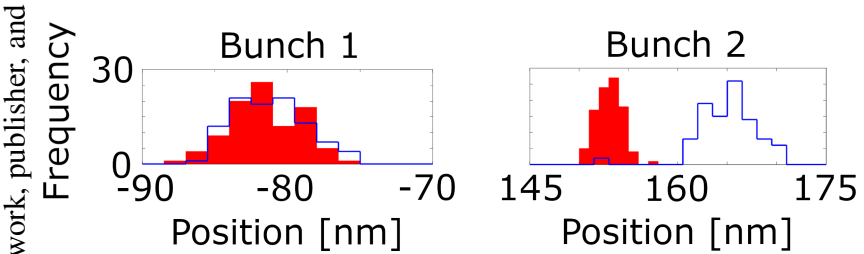

Figure 5: Predicted distribution of position at the focal point (calculated from the position at P2 and P3) with feedback off (outline) and feedback on (filled).

a laser beam in two and then crossing the two halves at the I IP to form a fringe pattern in the beam focal plane. The size of the fringes is given by $d=\lambda / 2 \sin (\theta / 2)$, where $\lambda$ is 8 the laser wavelength and $\theta$ is the crossing angle of the two laser paths. Laser photons are inverse Compton scattered by the electron beam and measured downstream of the IP. The position of the fringes relative to the beam is scanned Ey phase shifting one of the laser beams and the degree of variation of the scattered photon signal is quantified as a $\Xi$ modulation depth $(M)$, from which the vertical beam size $(\sigma)$ can be estimated. In addition to its application as a direct means of achieving the beam stability goal at ATF2, the FONT beam orbit feedback system has also been observed to cause a reduction in the apparent beam size at the IP [17]. The interaction of the electromagnetic field surrounding a o bunch of charged particles with geometrical discontinuities in the beamline results in wakefields. Each particle in the bunch receives a transverse deflection from the wakefield induced in the beam pipe by the passage of the preceding particles, leading to both a change in the measured orbit of $\dot{\overrightarrow{-}}$ the bunch as a whole as the center of mass shifts and a change ऽิ in the orbit of the tail of the bunch relative to the head. As the (2) IPBSM effectively measures the size of the distribution of particles at the IP integrated over many bunches, any increase in the beam position jitter or distortion of the transverse profile of the bunch is perceived as an increase in beam size. ATF2 is known [18] to be particularly sensitive to wakefields due to the long bunch length and the relatively low beam energy. The primary sources of wakefields in the ATF2 beamline are C-band cavity BPMs, bellows and vacto uum flanges [19]. The orbit change caused by wakefields at ATF2 has been reported [20] and several of the cavity BPMs were removed in order to reduce it. As the magnitude of the wakefield kick is proportional to the position offset $\overrightarrow{\bar{t}}$ between bunch and wakefield source (for small offsets), a $\Xi$ position feedback that reduced the offset between bunch and I wakefield source would be expected to mitigate the increase in beam size due to wakefields.

Figure 6a shows the beam size as a function of the beam charge when the beam was operated in two bunch mode with a bunch separation of $302.4 \mathrm{~ns}$. The vertical beam size $(\sigma)$ can be expressed as a function of a charge dependence parameter $(w)$ via the relation $\sigma=\left(\sigma_{0}^{2}+w^{2} Q^{2}\right)^{1 / 2}$ where $\sigma_{0}$ is the beam size in the absence of wakefields. Using the ATF2 cavity BPMs, the uncorrected vertical angle jitter of the second bunch at the IP was estimated to be $\sim 215 \mu \mathrm{rad}$
(Fig. 6b). With the upstream feedback system active, the jitter is reduced to $51 \mu \mathrm{rad}$. Figure 6a shows the measured size of the second bunch as a function of beam charge, both with and without feedback. It can be seen that stabilizing the position and angle of the second bunch with the FONT feedback system also reduced the charge dependence of the beam size measured at the IP by a factor of $1.6 \pm 0.2$, from $27.4 \pm 1.9 \mathrm{~nm} / 10^{9} e^{-}$to $16.9 \pm 1.6 \mathrm{~nm} / 10^{9} e^{-}$. The magnitude of this reduction is in line with what would be expected from a detailed model of beam transport in the ATF2 beamline including explicitly the known wakefield sources; full details are reported in [18-22].
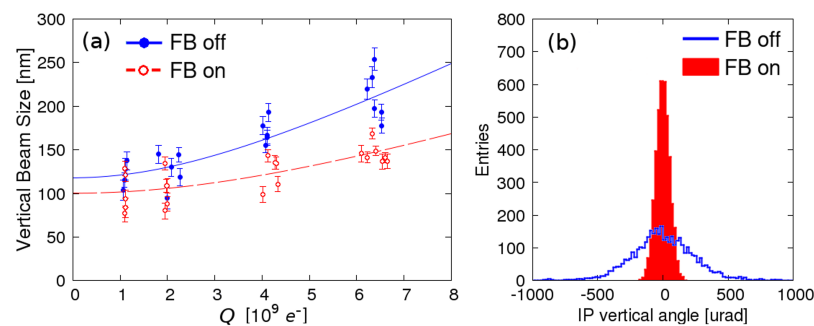

Figure 6: Beam size as a function of beam charge (left) and distribution of IP vertical angle jitter (right) for two bunch operation with feedback on (unfilled points, dashed line) and feedback off (filled points, solid line). Each point represents a single beam size measurement.

\section{CONCLUSIONS}

An intra-train position and angle feedback system has been developed to achieve the ATF2 beam stability goal Operating on a train of two bunches separated by $187.6 \mathrm{~ns}$, the feedback system stabilized the position of the second bunch at the feedback BPMs to the 270-340 nm level and the angle to within $250 \mathrm{nrad}$. The model of the beamline predicts that this level of correction would reduce the jitter at the focal point to $1.2 \pm 0.1 \mathrm{~nm}$, meeting the target beam stability performance. The potential of the feedback system towards reaching the beam size goalwas also measured. Scanning the charge of a beam consisting of two bunches separated by $302.4 \mathrm{~ns}$ showed that the feedback system reduced the charge dependence of the quadrature growth in beam size from $27.4 \pm 1.9 \mathrm{~nm} / 10^{9} e^{-}$to $16.9 \pm 1.6 \mathrm{~nm} / 10^{9} e^{-}$.

\section{REFERENCES}

[1] B. Grishanov et al., "ATF2 Proposal", SLAC, Menlo Park, USA, Rep. SLAC-R-771, 2005.

[2] B. Grishanov et al., "ATF2 Proposal Vol. 2", SLAC, Menlo Park, USA, Rep. SLAC-R-796, 2006.

[3] P. Bambade et al., "Present status and first results of the final focus beam line at the KEK Accelerator Test Facility", Phys. Rev. ST Accel. Beams vol. 13, p. 042801, 2010. doi : 10.1103/PhysRevSTAB. 13.042801

[4] G. White et al., "Experimental Validation of a Novel Compact Focusing Scheme for Future Energy-Frontier Linear Lepton Colliders", Phys. Rev. Lett., vol. 112, p. 034802, 2014. doi : /10.1103/PhysRevLett.112.034802

MC1: Circular and Linear Colliders 
[5] R. Apsimon et al., "Design and operation of a prototype interaction point beam collision feedback system for the International Linear Collider", Phys. Rev. Accel. Beams, vol. 21, p. 122802, 2018.

doi : 10.1103/PhysRevAccelBeams . 21. 122802

[6] P. Burrows et al., "Progress towards Electron-beam Feedback at the Nanometre Level at the Accelerator Test Facility (ATF2) at KEK", in Proc. 6th International Particle Accelerator Conference (IPAC'15), Richmond, VA, USA, May 2015, paper MOPTY083, pp. 1133-1135.

[7] R. Apsimon et al., "Design and performance of a high resolution, low latency stripline beam position monitor system”, Phys. Rev. ST Accel. Beams, vol. 18, p. 032803, 2015. doi : 10.1103/PhysRevSTAB . 18.032803

[8] N. Blaskovic Kraljevic et al., "Optimisation of a HighResolution, Low-Latency Stripline Beam Position Monitor System for Use in Intra-Train Feedback", in Proc. 8th International Particle Accelerator Conference (IPAC'17), Copenhagen, Denmark, May 2017, paper TUPIK110, pp. 19791982.

[9] G. Christian et al., "A fast, custom FPGA-based signal processor and its applications to intra-train beam stablisation", in Proc. 11th International Workshop on Personal Computers and Particle Accelerator Controls (PCaPAC'16), Campinas, Brazil, Oct. 2016, paper FRFMPLCO05, pp. 137-140.

[10] N. Blaskovic Kraljevic et al., "Intra-Train Position and Angle Stabilisation at ATF Based on Sub-Micron Resolution Stripline Beam Position Monitors", in Proc. 5th International Beam Instrumentation Conference (IBIC'16), Barcelona, Spain, Sep. 2016, paper TUPG15, pp. 349-352.

[11] N. Blaskovic Kraljevic et al., "Bunch-by-bunch Position and Angle Stabilisation at ATF based on Sub-micron Resolution Stripline Beam Position Monitors", in Proc. 7th International Particle Accelerator Conference (IPAC'16), Busan, Korea, May 2016, paper THPOR034, pp. 3859-3861.

[12] P. Burrows et al., "Design, Testing and Performance Results of a High-resolution, Broad-band, Low-latency Stripline Beam Position Monitor System", in Proc. 6th International Particle Accelerator Conference (IPAC'15), Richmond, VA, USA, May 2015, paper MOPTY084, pp. 1136-1138.

[13] G. Christian et al., "A Sub-micron Resolution, Wide-band, Stripline BPM System for Driving Bunch-by-bunch Feed- back and Feed-forward Systems at ATF", in Proc. 5th International Particle Accelerator Conference (IPAC'14), Dresden, Germany, Jun. 2014, paper TUPME009, pp. 1358-1360.

[14] S. Jang et al., "The Development of C-Band Cavity Beam Position Monitor with a Position Resolution of Nano Meter", in Proc. 7th International Particle Accelerator Conference (IPAC'16), Busan, Korea, May 2016, paper THOAA02, pp. 3149-3151.

[15] T. Bromwich et al., "Performance of Nanometre-Level Resolution Cavity Beam Position Monitors at ATF2", in Proc. 9th International Particle Accelerator Conference (IPAC'18), Vancouver, Canada, Apr. 2018, paper TUZGBD5, pp. 12121214.

[16] T. Suehara et al., "A nanometer beam size monitor for ATF2", Nucl. Instrum. Methods Phys. Res. A, vol. 616, 2010. doi : $10.1016 /$ j.nima . 2010.02 .065

[17] T. Okugi et al., "Beam jitter reduction with FONT intra-train feedback at ATF2", in Proc. 13th Annual Meeting of Particle Accelerator Society of Japan (PASJ2016), Busan, Korea, May 2016, paper MOOL04, pp. 34-38.

[18] P. Korysko, "Intensity-dependent effects in the Accelerator Test Facility 2 and extrapolation to future electron-positron linear colliders", Ph.D. thesis, Phys. Dept University of Oxford, Oxford, UK, 2020.

[19] T. Okugi et al., "Intensity dependence of ATF2 virtual IP beam size", in Proc. 16th Annual Meeting of Particle Accelerator Society of Japan (PASJ2019), Kyoto, Japan, 2019, paper FRPI023, pp. 1078-1081.

[20] J. Snuverink et al., "Measurements and simulations of wakefields at the Accelerator Test Facility 2", Phys. Rev. Accel. Beams, vol. 19, p. 091002, 2016. doi : 10.1103/PhysRevAccelBeams. 19.091002

[21] Y. Kim et al., "Cavity beam position monitor system for the Accelerator Test Facility 2", Phys. Rev. ST Accel. Beams, vol. 15, p. 042801, 2012. doi : 10.1103/PhysRevSTAB . 15.042801

[22] P. Korysko et al., "Wakefield effects and mitigation techniques for nanobeam production at the KEK Accelerator Test Facility 2", Phys. Rev. Accel. Beams, vol. 23, p. 121004, 2020. doi : 10.1103/PhysRevAccelBeams . 23.121004 